\title{
Postoperative neonatal mortality prediction using superlearning
}

\section{Jennifer N. Cooper, PhD'12; Peter C. Minneci, MD, MHSc ${ }^{134}$; Katherine J. Deans, MD, MHSc ${ }^{134}$}

${ }^{1}$ Center for Surgical Outcomes Research and the Center for Innovation in Pediatric Practice, The Research Institute at Nationwide Children's Hospital, ${ }^{2}$ Department of Pediatrics, The Ohio State University College of Medicine, ${ }^{3}$ Department of Surgery, Nationwide Children's Hospital, ${ }^{4}$ Department of Surgery, The Ohio State University College of Medicine

Introduction

With the current availability of large electronic medical datasets, it is
possible to develop robust clinical prediction models that enable clinis to accurately estimate individual patient's risks of morbidity and mortality Traditionally, logistic regression models have been used to derive these predictions Logistic regression imposes stringent, parametric constraints on the $\cdot \log (p /(1-p))=\beta_{0}+\beta_{1} X_{1}+\beta_{2} X_{2}+\ldots+\beta_{p} X_{p}$ Superlearning is an ensemble machine learning method for selecting via cross-y
a set 1

Superlearning relies on an analyst-speciified collection of algorithms and performance measure

- The objective of this study was to develop and validate a clinical prediction model for 30 -day postoperative mortality in neonates using

\section{Methods}

- Used 2012-14 National Surgical Quality Improvement Program-Pediatic Patients treated in 2012-13 formed the development sample ( $N=6499$, $3.6 \%$ mortality), and those treated in 2014 formed the validation samp

- Used 211 preoperative predictors, 14 algorithms and 10-fold cross

-2 stepwise logistic regression models, 3 penalized logistic regression
models, 2 generalized boosted regression models, 5 random forest models, 2 generalized boosted regress
models, and 2 classification tree models

- Repeated analysis after screening out predictors with $p>0.20$ in bivariate

Examined discrimination (AUROC) and calibration (calibration intercep and slope) of superlearner and all constituent algorithms in both the

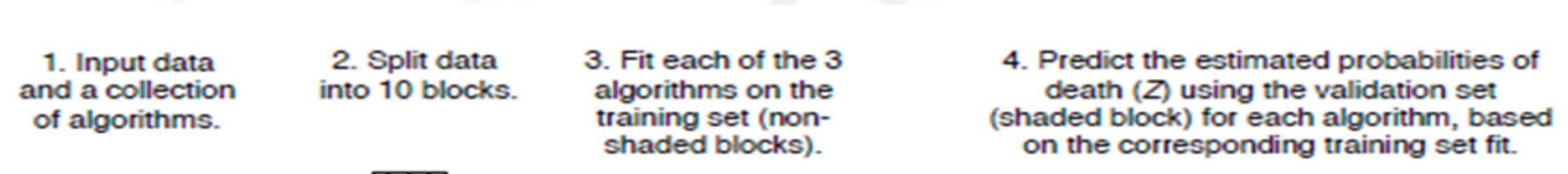

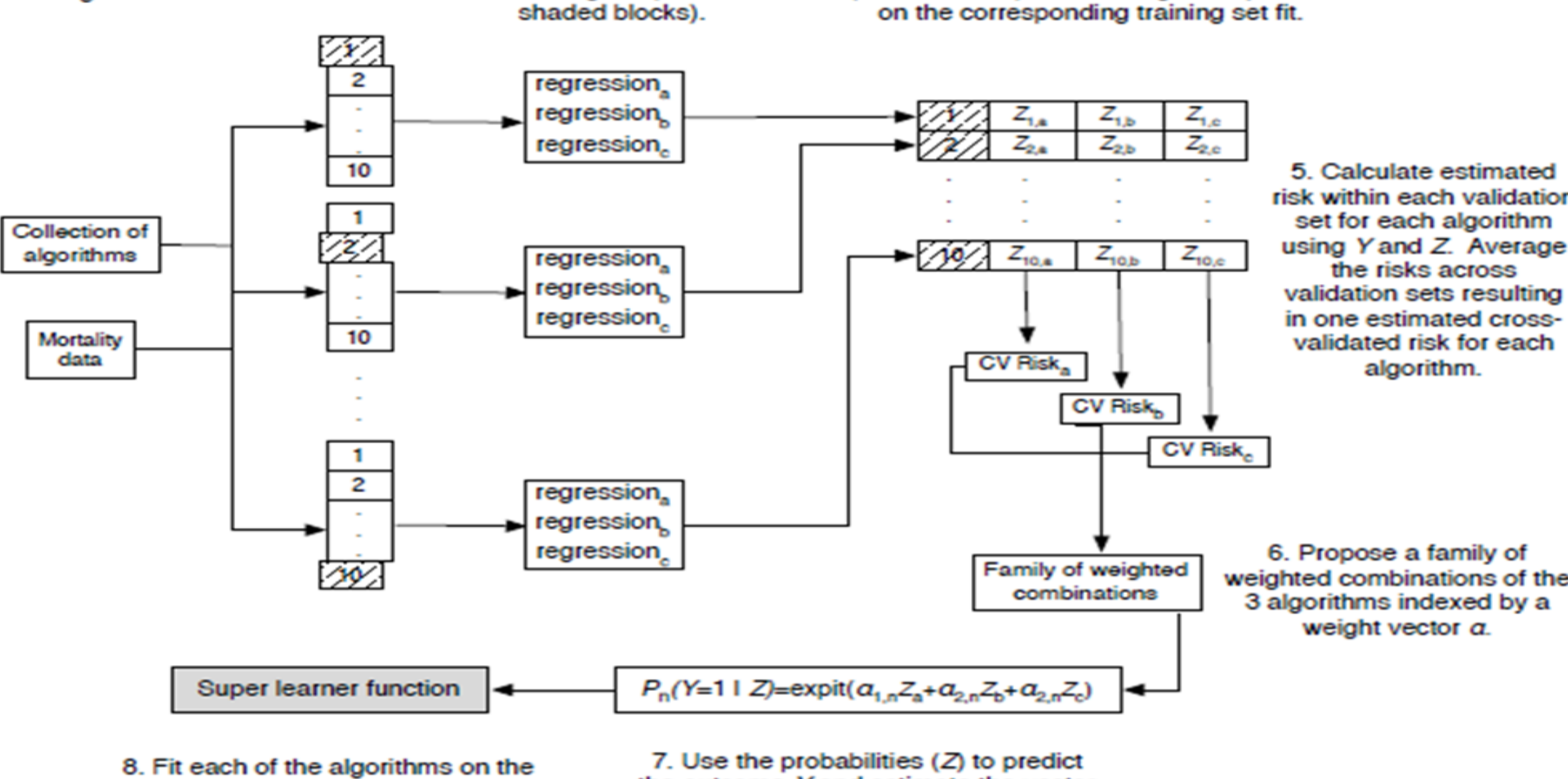

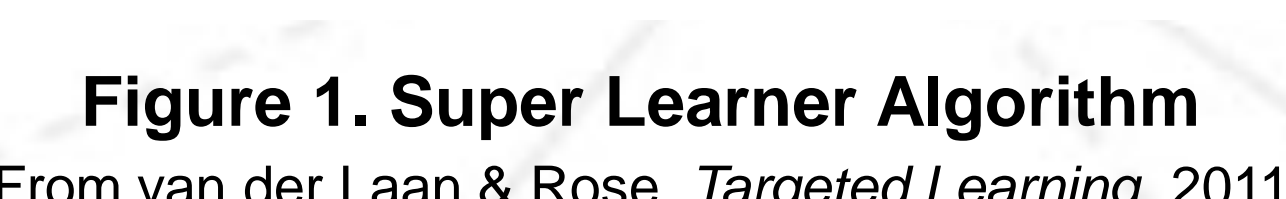

Table 1. Patient characteristics at the time of surgery
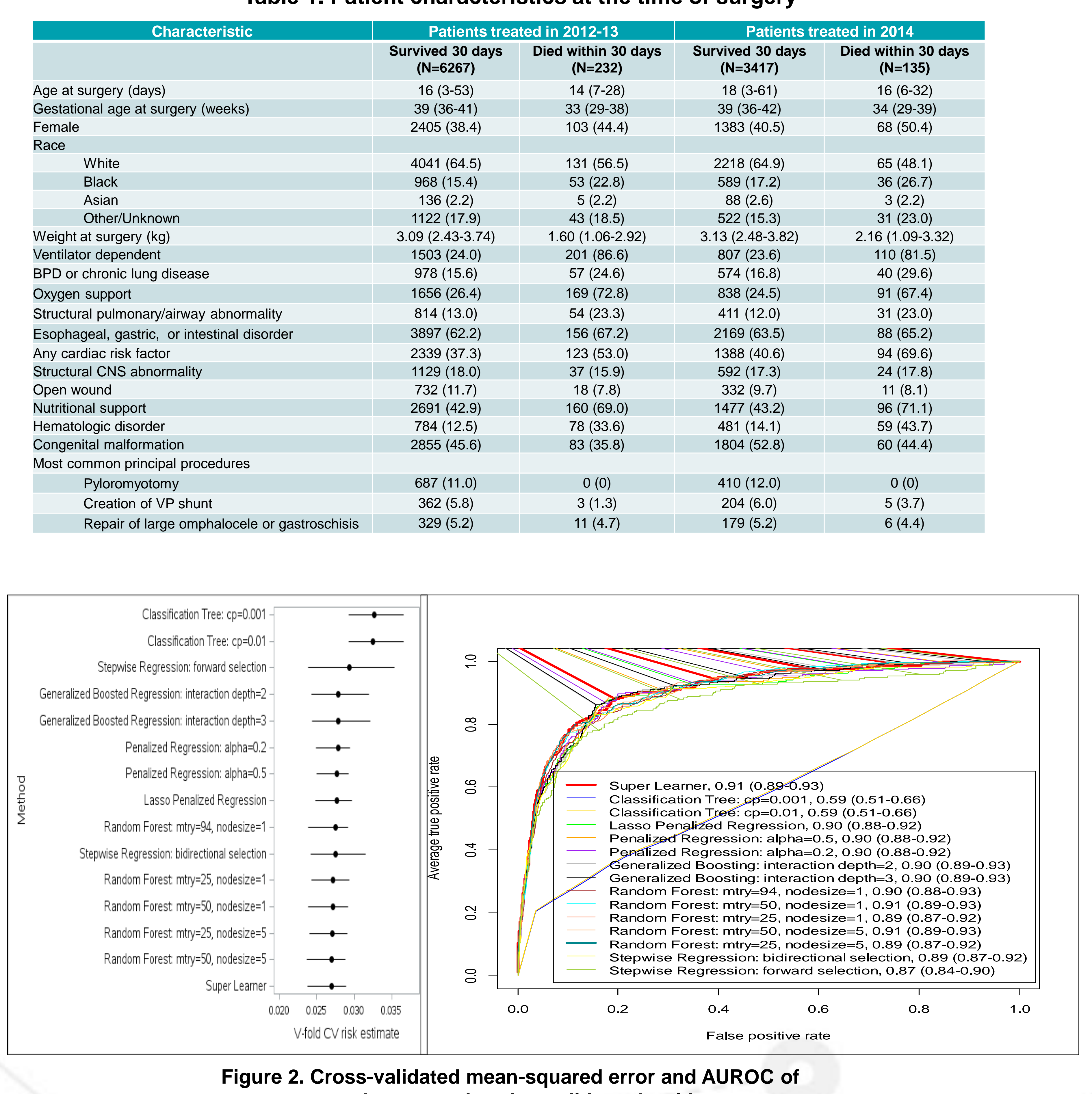

Figure 2. Cross-validated mean-squared error and AU
super learner and each candidate algorithm

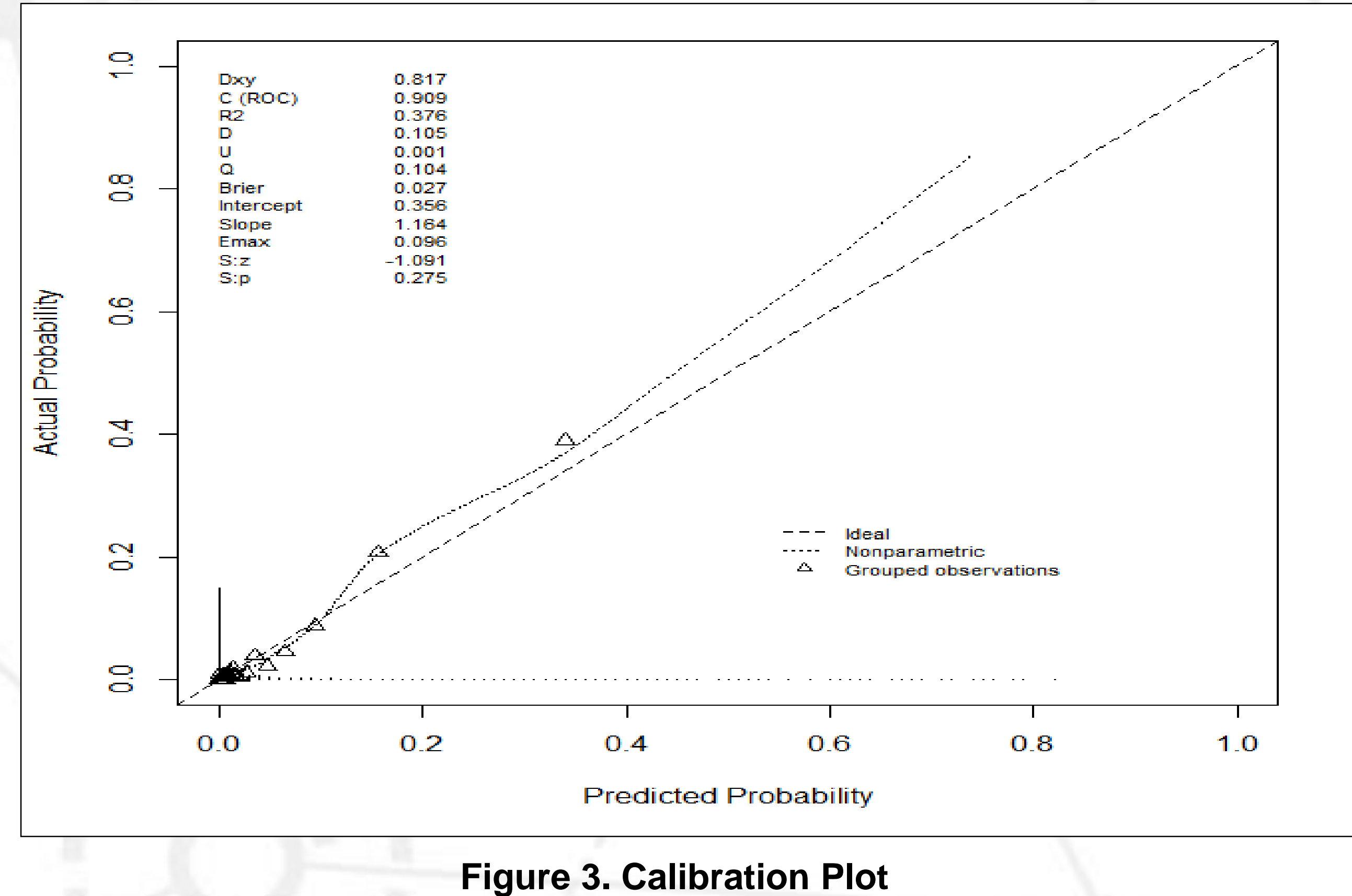

- The super learner showed good discrimination but poor calibration in the validation sample: AUROC: 0.87 (95\% Cl $0.83-0.90)$
Results

Table 2. Mortality predictions obtained from super learner for two different patient profiles

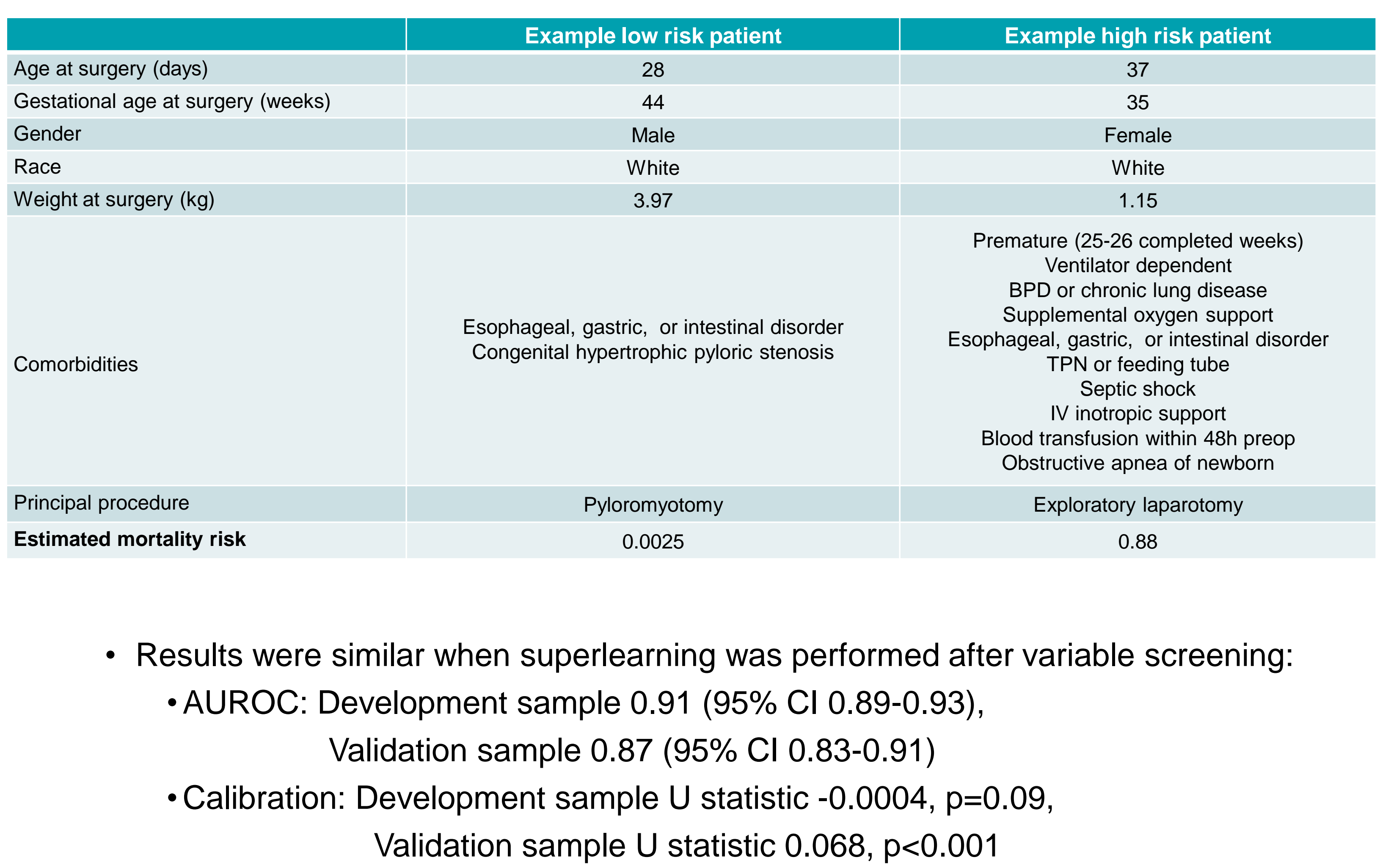

Conclusions

- Superlearning provided improved or equivalent accuracy when compared to individual regression an machine learning algorithms for the
calibration in a validation sample

Though many studies have reported algorithms for predicting neonatal mortality after particular types of surgical procedures, the vast majority have used main effects logistic regression modeling - The Super Learner ofters a flexible alternative to other non-parametric methods because it can
include as many candidate algorithms as desired, and will perform at least as well as the best individual algorithm in its library

- Super learning should be considered for prediction in large datasets whenever complex mechanisms make parametric modeling assumptions unrealistic

- Alhough the Super Leanter wil perform no worse than he best constituent algortithm in a training

- Poor calibration in our validation data may have been due to 11 new hospitals joining the NSQIP-

References

1. van der Laan MJ, Polley EC, Hubbard AE. Super learner. Stat Appl Genet Mol Biol 2007;6:25. 2. Polley EC, van der Laan MJ. SuperLeaner: Super Learner Prediction. 2014; version 20-15: Available from: https://github.com/ecpolley/SuperLearner.

\section{Acknowledgements}

The American College of Surgeons National Surgical Quality Improvement Program and the hospitals The American College of Surgeons National Surgical Quality Improvement Program and the hospitals
participiating in the ACS NSSIP Pediatric are the source of the data used herein; they have not verified
and are not responsible for the statistical validity of the data analysis or the conclusions derived by the
authors. 